\title{
Erst- und Weiterbildung und die europäische Bildungspolitik
}

\author{
Jutta Allmendinger \\ Christian Ebner
}

\begin{abstract}
Als sich im Jahr 2000 der Europäische Rat in Lissabon das Ziel setzte, „die Europäische Union zum wettbewerbsfähigsten und dynamischsten wissensbasierten Wirtschaftsraum der Welt zu machen", war allen klar, wie dies zu erreichen sei. Bildung und Weiterbildung waren die definierten Pfade - und sind es heute noch. Erst jüngst, Ende 2006, wurde dies nochmals unterstrichen, als die Notwendigkeit zu „lebenslangem Lernen“ in einem Beschluss des Europäischen Parlaments und des Rates hervorgehoben und entsprechende finanzielle Mittel zur Verfügung gestellt wurden: Von 2007 bis 2013 stehen für dieses Projekt fast $7 \mathrm{Mrd}$. $€$ bereit. Europäisches Engagement für verstärkte (Weiter-)Bildungsbemühungen ist dringend geboten und längst überfällig. Dies gilt gerade für Deutschland, das überdurchschnittlich stark auf hochqualifizierte Personen angewiesen ist. Niedrigqualifizierte finden hierzulande kaum noch Jobs, wie entsprechende Arbeitslosenquoten belegen: Lag die Arbeitslosenquote von Akademikern im Jahr 2005 bei rund 4,1\%, war sie für Personen ohne abgeschlossene Berufsausbildung mehr als 6-mal so hoch (26\%). Lehr- und Fachschulabsolventen reihen sich hinsichtlich der Arbeitsmarktchancen in die Mitte ein, bei einer Arbeitslosenquote
\end{abstract} von $9,7 \%$.

Doch für viele Jugendliche ist schon die Aufnahme einer beruflichen Lehre schwierig. Von den rund 734.000 bei der Bundesagentur für Arbeit gemeldeten Lehrstellenbewerbern hat im Laufe des Ausbildungsjahres 2007 (bis Ende September) weniger als die Hälfte, etwa 320.000 , eine Berufsausbildung im dualen System begonnen. Und über die Hälfte der Bewerber hat die Schule schon ein oder mehrere Jahre früher verlassen. Salopp nennt man diese Jugendlichen „Altbewerber". Was geht hier vor, in einer konjunkturell doch guten Zeit? Zum einen mangelt es in der Tat an einem ausreichenden Angebot an Lehrstellen. Doch hinzu kommt auch, dass manche Lehrstellen nicht besetzt werden können, den Jugendlichen mangelt es schlicht an "Ausbildungsreife“. Dies heißt aber auch, dass der erwartete Fachkräftemangel zu wesentlichen Teilen hausgemacht ist. Man investiert zu wenig in Ausbildung und achtet vorher viel zu wenig auf eine gute Bildung. Noch immer wird zu selten präventiv während der Schul- und Vorschulzeit dafür gesorgt, Bildungsbenachteiligungen entgegenzusteuern und Bildungsarmut gar nicht erst entstehen zu lassen. Das unterscheidet uns von einigen anderen Ländern.

Auch um das lebenslange Lernen steht es schlecht, Weiterbildungsaktivitäten sind in Deutschland im internationalen Vergleich eher selten. Wie der OECD-Band „Bildung auf einen Blick“berichtet, nehmen im Laufe eines Jahres $12 \%$ der Deutschen an einer berufsbezogenen Weiterbildung teil. Dies liegt deutlich unter der durchschnittlichen Teilnahmequote in der OECD (18\%) und ist verglichen mit Ländern wie Dänemark (39\%) oder Schweden (40 \%) niedrig. Hinzu kommt, dass Weiterbildung ganz ungleich verteilt ist: Gering ausgebildete Personen nehmen fast nie, gut ausgebildete deutlich häufiger daran teil. Der Teufelskreis setzt sich fort. Wir sind "toploaded“. Und auch hier zeigt sich, dass Weiterbildung zu einem großen Teil erst dann betrieben wird, wenn es zu spät ist, also Arbeitslosigkeit vorliegt. Wir sind „emergency-loaded“. Von einem vorsorgenden, investiven Sozialstaat kann keine Rede sein. Auch nicht von einer Lebensverlaufspolitik, die ernst nimmt, dass mancher länger arbeiten muss und will. Wir sind im Zeitverlauf "front-loaded“: Denn in Deutschland bleibt Weiterbildung an die jungen Jahre gebunden, nach dem Alter von 50, erst recht nach dem Alter von 55 Jahren, tut sich wenig. Doch selbst dann sollen Personen noch zwölf Jahre lang erwerbstätig sein. Wie scheinheilig ist auch in diesem Zusammenhang das Jammern über den drohenden Fachkräftemangel. Kurzum, in allen drei Dimensionen hat unsere Weiterbildungspolitik eine Ausrichtung, die systematisch am Bedarf vorbeigeht.

Bei all dem ist zu bedenken, dass wir jenseits der Statistiken der Bundesagentur für Arbeit so genau gar nicht wissen, was eigentlich hinter einer „Weiterbildung“ steckt. Häufig dürften dies zweiwöchige abendliche Softwarekurse sein. In den allerseltensten Fällen hingegen längerfristig angelegte Fortbildungen oder auch Umschulungen hin zu neuen Tätigkeitsformen für die späteren Erwerbsjahre. Auf diese Weise werden wir den Problemen von Maurern, Dachdeckern und allen anderen körperlich hart arbeitenden Menschen nie begegnen und können wir keinen Grundstein zu längerer Beschäftigung legen. Weiterbildung als Beruhigungsmittel, das reicht heute nicht mehr.

Die von der EU geforderte und (finanziell) geförderte Politik eines deutlichen „Mehr" an Bildung und Weiterbildung muss auf nationaler Ebene ausreichend flankiert werden, sie kann sonst hier gar nicht Fuß fassen. Der jüngste Vorstoß der Bundesregierung, die Bezugsdauer des Arbeitslosengeldes I für Ältere zu verlängern und nicht, wie es an sich nötig wäre, in deren Weiterbildung zu investieren, setzt am falschen Punkt an. Diese Politik, die auf das Fördern verzichtet, vermittelt doch wieder die Botschaft, dass man immer noch früh in den Ruhestand eintreten kann. Und sie verhindert auch, dass ein gutes nationales Lernklima entstehen kann.

\footnotetext{
Jutta Allmendinger, Präsidentin des Wissenschaftszentrums Berlin für Sozialforschung und Professorin für Bildungssoziologie und Arbeitsmarktforschung an der Humboldt-Universität zu Berlin. e-mail: Jutta.Allmendinger@wzb.eu

Christian Ebner, Doktorand in der Projektgruppe "Education and Transitions into the Labour Market" am Wissenschaftszentrum Berlin für Sozialforschung. e-mail: Christian.Ebner@wzb.eu
} 